\title{
Le complexe glaciaire du Cap Charles, vallée moyenne du
} Saint-Laurent, Québec

The Cap Charles Glacial Complex of the Middle St. Lawrence

Valley, Québec

\section{Der glaziale Komplex von Cap Charles, mittleres Sankt lorenz-Tal, Québec}

\section{François Bernier et Serge Occhietti}

Volume 44, numéro 2, 1990

URI : https://id.erudit.org/iderudit/032816ar

DOI : https://doi.org/10.7202/032816ar

Aller au sommaire du numéro

\section{Éditeur(s)}

Les Presses de l'Université de Montréal

\section{ISSN}

0705-7199 (imprimé)

1492-143X (numérique)

Découvrir la revue

\section{Citer cet article}

Bernier, F. \& Occhietti, S. (1990). Le complexe glaciaire du Cap Charles, vallée moyenne du Saint-Laurent, Québec. Géographie physique et Quaternaire, 44(2), 173-180. https://doi.org/10.7202/032816ar

\section{Résumé de l'article}

Le complexe du Cap Charles est emboîté dans les rythmites de Leclercville, sur la rive sud du Saint-Laurent, en aval de Deschaillons. Il est composé d'une lithozone inférieure de till de fond (diamicton I) dont la base incorpore les argiles des rythmites de Leclercville et dont la fabrique indique un écoulement glaciaire vers le SE. Au-dessus, une lithozone de sables turbiditiques, puis deux zones de till flué (diamictons II et III), séparées par une zone d'argile stratifiée, représentent une sédimentation de marge glaciaire en milieu lacustre. Le sommet est une surface d'érosion. Les turbidites de Parisville à lits diamictiques couvrent le complexe du Cap Charles et précèdent le Till de Gentilly. Faute de repères, la position stratigraphique du complexe du Cap Charles est incertaine. Il représente soit des phases préliminaires à l'englaciation majeure représentée par le Till de Gentilly, postérieures à l'Intervalle de Saint-Pierre, soit des phases associées aux tills immédiatement antérieurs à l'Intervalle de Saint-Pierre. Il a été mis en place par des glaces allochtones venant des Laurentides puis par fluages, vraisemblablement à la périphérie d'un lobe. Le complexe du Cap Charles révèle que l'histoire glaciaire de la vallée du Saint-Laurent est complexe et reste encore peu connue. 


\section{LE COMPLEXE GLACIAIRE DU CAP CHARLES, VALLÉE MOYENNE DU SAINT-LAURENT, QUÉBEC*}

François BERNIER et Serge OCCHIETTI, respectivement Département des sciences de la Terre et Département de géographie et GEOTOP, Université du Québec à Montréal, C.P. 8888, succursale A, Montréal, Québec H3C 3P8.

RÉSUMÉ Le complexe du Cap Charles est emboîté dans les rythmites de Leclercville, sur la rive sud du Saint-Laurent, en aval de Deschaillons. II est composé d'une lithozone inférieure de till de fond (diamicton I) dont la base incorpore les argiles des rythmites de Leclercville et dont la fabrique indique un écoulement glaciaire vers le SE. Au-dessus, une lithozone de sables turbiditiques, puis deux zones de till flué (diamictons II et III), séparées par une zone d'argile stratifiée, représentent une sédimentation de marge glaciaire en milieu lacustre. Le sommet est une surface d'érosion. Les turbidites de Parisville à lits diamictiques couvrent le complexe du Cap Charles et précèdent le Till de Gentilly. Faute de repères, la position stratigraphique du complexe du Cap Charles est incertaine. II représente soit des phases préliminaires à l'englaciation majeure représentée par le Till de Gentilly, postérieures à l'Intervalle de SaintPierre, soit des phases associées aux tills immédiatement antérieurs à l'Intervalle de Saint-Pierre. II a été mis en place par des glaces allochtones venant des Laurentides puis par fluages, vraisemblablement à la périphérie d'un lobe. Le complexe du Cap Charles révèle que I'histoire glaciaire de la vallée du Saint-Laurent est complexe et reste encore peu connue.
ABSTRACT The Cap Charles glacial complex of the middle St. Lawrence Valley, Québec. The Cap Charles glacial complex is imbricated into the Leclercville rhythmites, on the southern shore of the St. Lawrence River. This complex comprises three diamictons. Diamicton I is interpreted as a basal till with incorporated clay from the Leclercville rhythmites in the lower zone and with a SE trending fabric. Diamicton I is covered by a sandy turbidite lithozone. Diamictons II and III are flow tills separated by a stratified clay. As a whole the glacial complex results from ice marginal sedimentation in a lacustrine environment. The top of this unit is an erosional unconformity. The Parisville turbidites cover the Cap Charles complex and are themselves overlaid by the Gentilly Till. Because of the lack of marker beds, the stratigraphic position of the Cap Charles glacial complex is uncertain. It is either younger than the St-Pierre Sediments and would represent early glacial phases of the main Gentilly Till episode, or it is older than the St-Pierre Sediments and therefore would be associated with the tills laid down before the St. Pierre interval. Nevertheless, the Cap Charles complex indicates an allochtonous ice invasion from the Laurentide Highlands and a likely local ice flow at the periphery of an ice lobe. The glacial history of the middle St. Lawrence Valley is still poorly known and seems to be complex.
ZUSAMMENFASSUNG Der glaziale Komplex von Cap Charles, mittleres Sankt lorenz-Tal, Québec. Der komplex von Cap Charles ist in die Rythmite von Leclercville eingefügt, auf dem Südufer des Sankt Lorenz, flussabwärts von Deschaillons. Er besteht aus einer unteren Lithozone von Grundtill (Diamikton I), deren Basis Ton aus den Rytmiten von Leclercville enthält und deren Machart auf einen glazialen Abfluss nach SO hinweist. Darüber repräsentieren eine Lithozone aus schlammigem Sand und dann zwei Zonen von geflossenem Till (Diamikton II u. III), welche durch eine Zone geschichteten Tons voneinander getrennt sind, eine Sedimentierung des glazialen Rands in einem Seemilieu. Die Spitze ist eine Erosionsoberfläche. Die Trübungen von Parisville mit diamiktischen Betten bedecken den Komplex von Cap Charles und werden ihrerseits von dem Till von Gentilly bedeckt. Da es keine Anhaltspunkte gibt, ist die stratigraphische Position des Cap CharlesKomplex unsicher. Er stellt entweder Vorphasen der bedeutenden Vereisung, die durch das Till von Gentilly repräsentiert wird und welche auf das Intervall von Saint-Pierre folgten dar, oder Phasen, die mit den Tills unmittelbar vor dem Intervall von Saint-Pierre in Verbindung gebracht werden. Er ist durch allochtones Eis von den Laurentides angelegt worden und dann durch Fliessen, wahrscheinlich an der Peripherie eines Eislappens. Der Komplex von Cap Charles lässt erkennen, dass die glaziale Geschichte des Sankt Lorenz-Tals komplex und noch wenig bekannt ist.

\footnotetext{
* Contribution du $6^{\mathrm{e}}$ Congrès de l'Association québécoise pour l'étude du Quaternaire, sous la direction de Bernard Hétu
} 


\section{INTRODUCTION}

L'état des connaissances sur la stratigraphie du Quaternaire de la vallée moyenne du Saint-Laurent a évolué très rapidement au cours des dernières années. Le schéma stratigraphique présenté par Gadd (1971), comprenant deux épisodes glaciaires séparés d'un intervalle non glaciaire, a subi plusieurs modifications. Trois épisodes glaciaires sont maintenant reconnus et sont représentés par les tills de Gentilly, du Cap Lévrard (Lamothe, 1987) et un till plus ancien à la base des sédiments quaternaires, sous le niveau du fleuve (Lamothe, 1987).

Au cap Charles (fig. 1), site naturel situé à 2,4 km en aval de Deschaillons sur la rive sud du Saint-Laurent, un complexe de trois diamictons d'origine glaciaire et une unité turbiditique, antérieurs au Till de Gentilly, reposent par-dessus des rythmites. Cet article présente ces nouvelles unités lithostratigraphiques. D'autres unités d'origine glaciaire antérieures au Till de Gentilly ont été identifiées dans la même région. La correspondance entre ces unités et le complexe du Cap Charles pose un problème stratigraphique; c'est pourquoi les noms des unités proposés dans ce texte sont informels mais en conformité avec le code stratigraphique international (Hedberg, 1979).

\section{CONTEXTE GÉOLOGIQUE GÉNÉRAL}

La région de Deschaillons-cap Charles est située sur la rive sud du Saint-Laurent et fait partie de la vallée moyenne du Saint-Laurent (fig. 1). Le substratum rocheux est composé de schistes et de calcaires ordoviciens des groupes de Black River, Trenton, Utica et Richmond (Clark et Globensky, 1975).

À l'emplacement même des coupes étudiées, le schiste d'Utica est la roche de fond. Malgré plusieurs travaux, notamment ceux de Keele (1915), Karrow (1957), Terasmae (1958), Gadd (1971), Occhietti (1982), Lamothe (1985), Occhietti et al. (1989), il reste encore beaucoup à faire pour établir de façon claire la stratigraphie du Quaternaire et reconstituer avec précision la succession des glacio-environnements de cette région. Les formations quaternaires constituent dans la région une couverture relativement épaisse, de sorte que l'on rencontre fréquemment en bordure du fleuve des coupes naturelles escarpées de l'ordre de 20 à $30 \mathrm{~m}$ de hauteur. Sous le niveau du fleuve, les sédiments meubles atteignent des épaisseurs relativement importantes, de l'ordre de 30 à $40 \mathrm{~m}$ d'après des forages de Simard (1971) et Lamothe (1987). A Leclercville, il y aurait au minimum de 15 à 20 m de dépôts sous le pied de la falaise selon des sondages sismiques (Vézina et Leroux, communication écrite).

\section{MÉTHODOLOGIE}

La coupe composite du cap Charles a été levée en 1987 et 1988. Elle comprend huit unités lithostratigraphiques (fig. 2) et est établie à partir de trois coupes principales, levées sur une distance de $200 \mathrm{~m}$. Dans ce texte, nous désignerons ces coupes d'amont en aval par les vocables Tennis 1, 2 et 3 . Les unités d'origine glaciaire sont définies selon les critères de Boulton (1968, 1970a, 1970b, 1976) et de Dreimanis (1976). Le code de lithofaciès de Eyles et al. (1983) a été utilisé pour décrire graphiquement les unités lithostratigraphiques. Pour effectuer les fabriques de till, nous n'avons mesuré que les cailloux dont le rapport des axes b / a et $c$ / b était plus petit que 0,67 (Parent 1987), ce qui correspond à des cailloux relativement plats et allongés. Des graphiques de contour de densité ont ensuite été obtenus avec l'aide du logiciel Stéréo de McEachran (1986). Pour chaque fabrique, au moins 50 cailloux ont été mesurés sur des coupes fraîchement dégagées. Des comptages lithologiques ont aussi été effectués dans chaque unité diamictique, sur une moyenne de 100 cailloux de $2 \mathrm{~cm}$ à $20 \mathrm{~cm}$ de diamètre.

\section{DESCRIPTION DES UNITÉS LITHOSTRATIGRAPHIQUES}

Au site du cap Charles, le substrat rocheux est sous le niveau du fleuve. Les unités de la coupe composite se décrivent ainsi (fig. 2):

Unité $n^{\circ} 1$ : rythmites de Leclercville

Une série de rythmites silto-argileuses compose cette unité. Elles affleurent sur près de 25 m d'épaisseur au-dessus du pied de la falaise à Leclercville, alors qu'au site du cap Charles elles n'apparaissent que sur $3,70 \mathrm{~m}$ au-dessus du niveau des marées hautes. La base de la coupe Tennis 3 a été nettoyée au tuyau d'arrosage alimenté par une pompe, ce qui nous a permis d'observer le contact entre les rythmites de Leclercville et le diamicton I du complexe du Cap Charles. Les rythmites sont faiblement inclinées entre 0 et $2,80 \mathrm{~m}$ au-dessus du niveau des marées hautes. Elles sont déformées entre 2,80 m et la base du diamicton I (3,70 m).

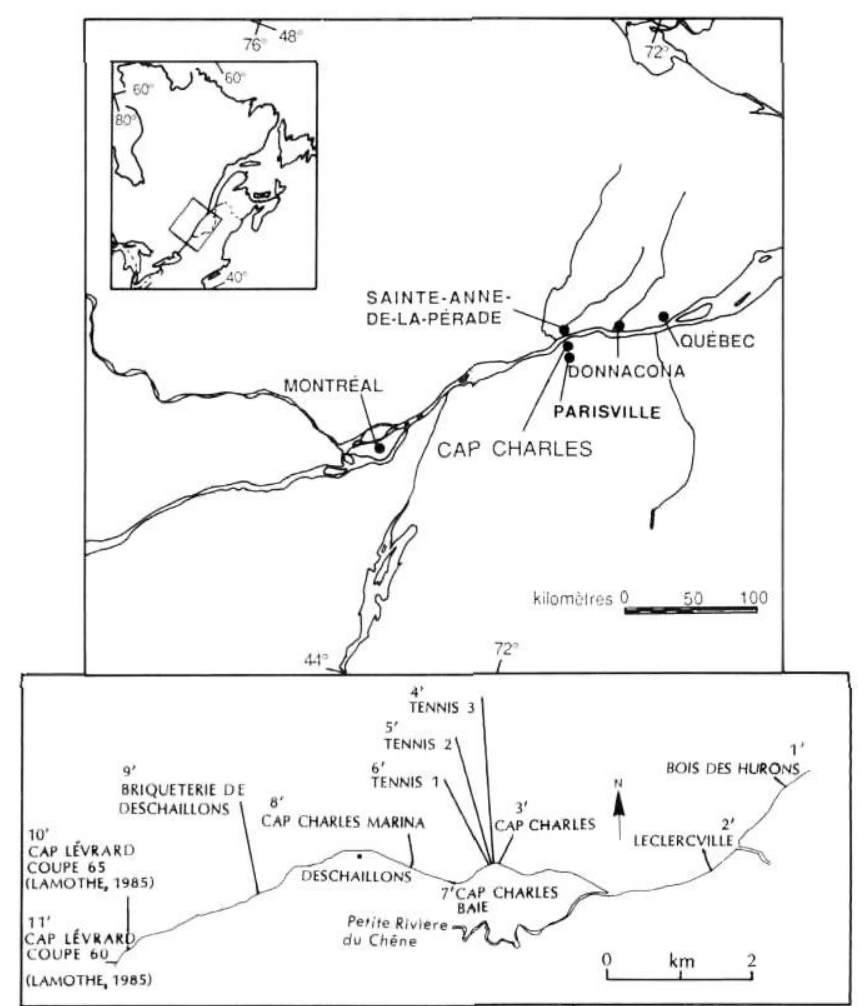

FIGURE 1. Localisation des sites mentionnés dans le texte. Location of sites referred to in the text. 


\section{COUPE COMPOSITE}

\section{CAP CHARLES}
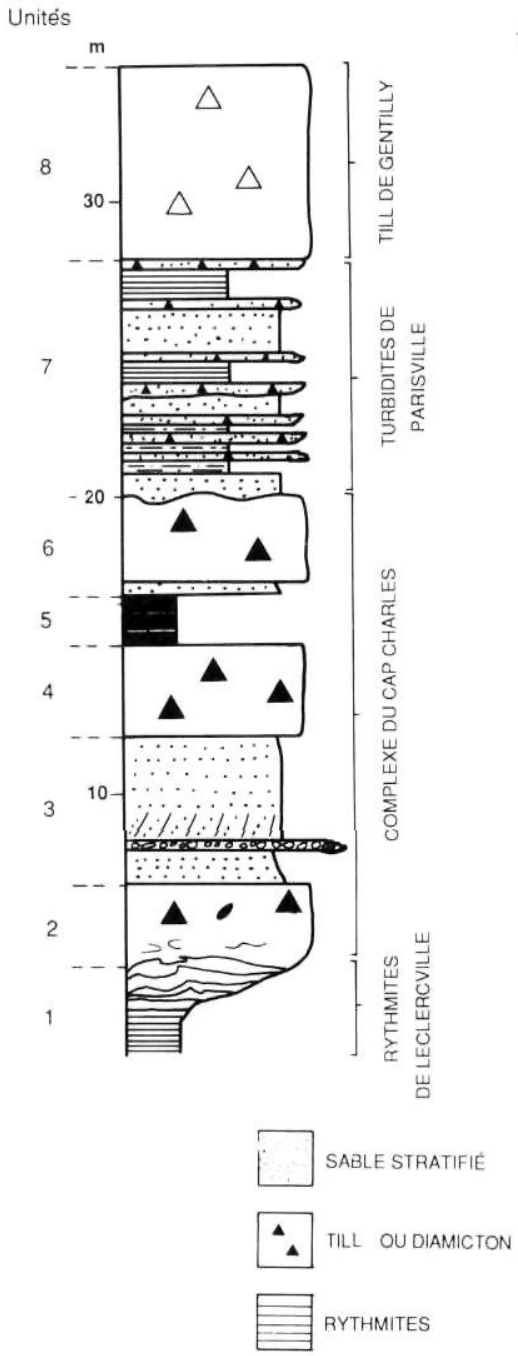

\section{DONNÉES \\ GRANULOMÉTRIQUES}

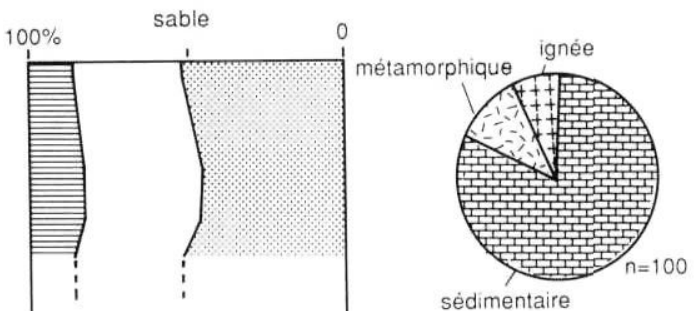

sédimentaire
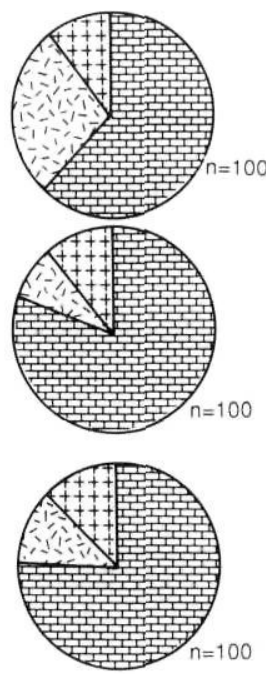

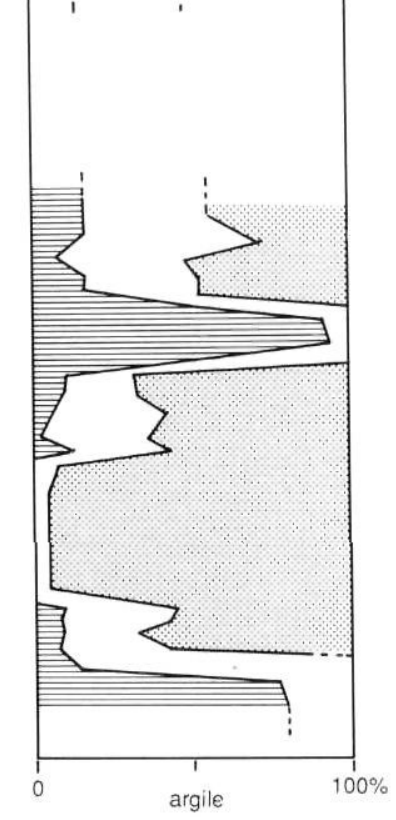

$\triangle \triangle$ TILL DE GENTILIY

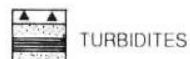

ARGILE LITEE

Unité $\mathrm{n}^{\circ} 2$ : diamicton I du complexe du Cap Charles

Cette unité, visible seulement sur la coupe Tennis 3, est un till constitué de matériaux hétérométriques à matrice siltosableuse dominante, dans laquelle les cailloux de forme glaciaire typique, c'est-à-dire en forme de chevron tronqué à une extrémité, sont abondants. D'une puissance de $3,5 \mathrm{~m}$, ce till est massif et compact. Humide, il est gris pâle à gris foncé. La fabrique indique une orientation des cailloux vers le SE, alors que le comptage lithologique montre une nette dominance $(78 \%)$ de roches sédimentaires, composées principalement de calcaire de Trenton. Paradoxalement, la teneur en carbonates de la matrice, de l'ordre de $3 \%$, est relativement faible (fig. 2). Les coefficients de classement et d'asymétrie de Folk et Ward (1957) indiquent que la matrice de ce sédiment est pauvrement classée et enrichie en particules fines $(<0,063 \mathrm{~mm})$, surtout à sa base, à cause de l'incorporation des argiles sous-jacentes (fig. 3). Une concrétion discoïdale striée, provenant soit des Varves de Deschaillons sous-jacentes, soit des varves plus anciennes de Deschambault (Occhietti et al.,

ANALYSES

DIRECTIONNELLES (FABRIQUES DE TILLS)
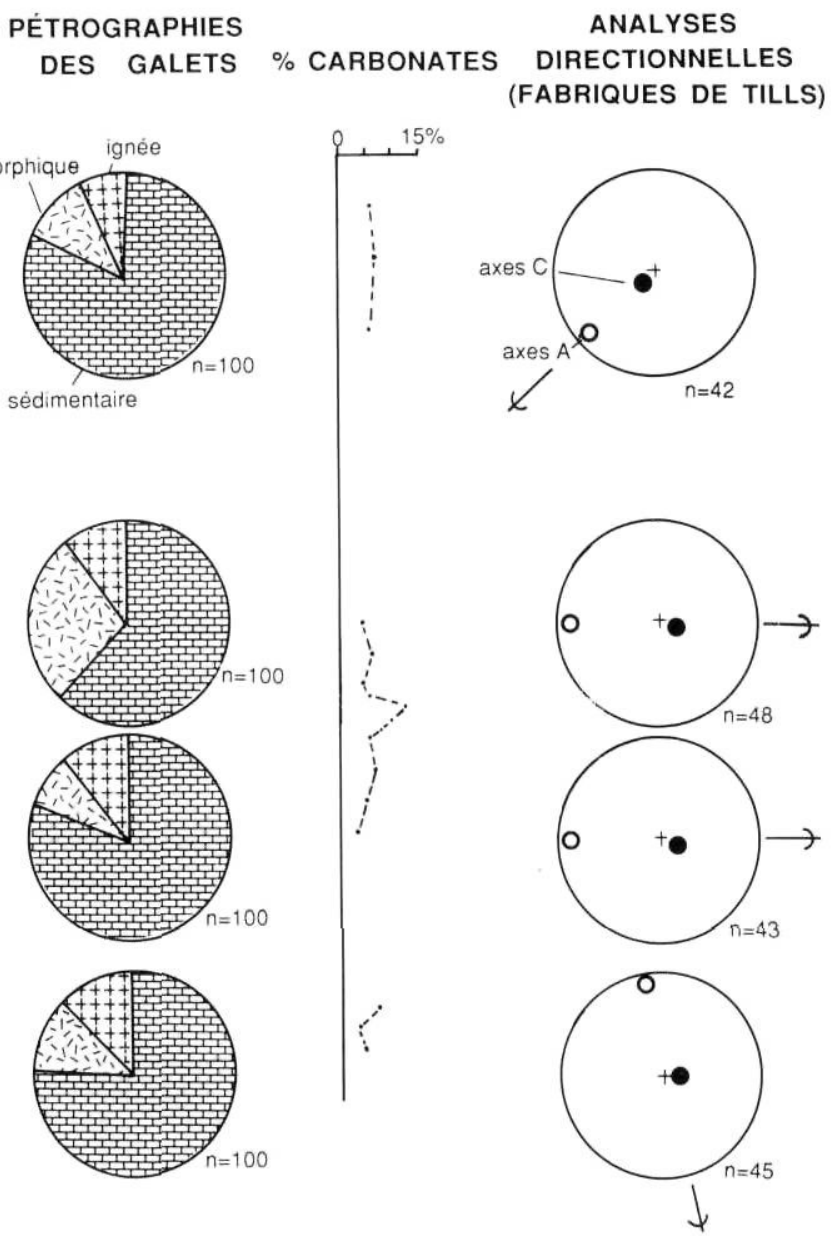

FIGURE 2. Colonne stratigraphique composite du site du complexe du Cap Charles.

Composite lithostratigraphic section of the Cap Charles glacial complex.

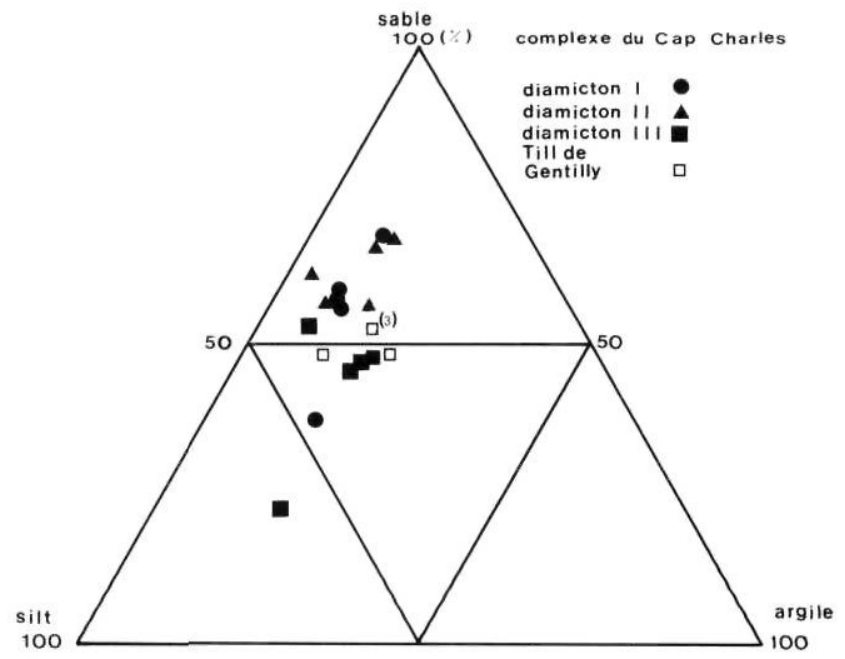

FIGURE 3. Résultats d'analyses granulométriques sur la matrice des diamictons I, II et III.

Grain size of the matrix of diamictons I, II and III. 

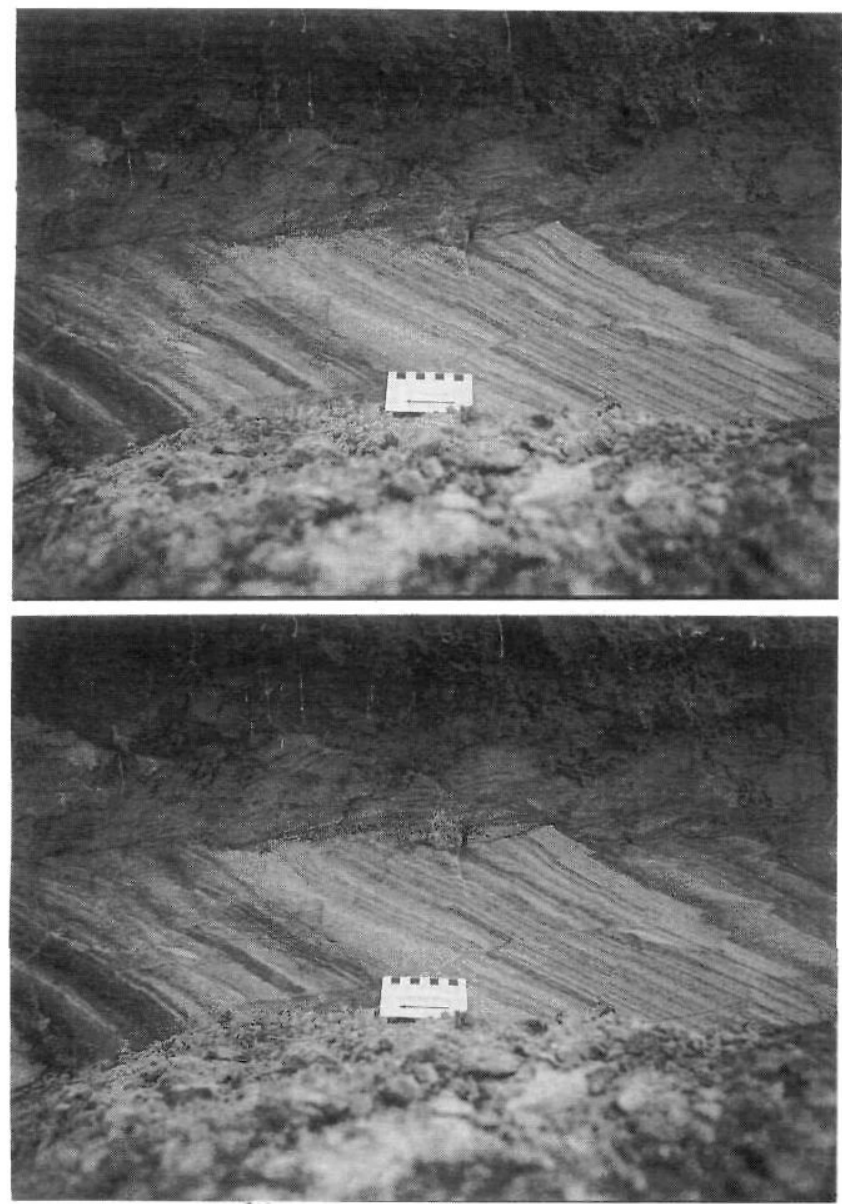

FIGURE 4. Sable à lits obliques et à failles inverses de la sous-unité 3a.

Faulted and cross-bedded sand of unit $3 a$.

1989) ou des rythmites sous le niveau du fleuve, a été trouvée dans cette unité. Des lambeaux de rythmites de Leclercville sont incorporés à la base du till. Des plans de cisaillement subhorizontaux tranchent la partie supérieure de l'unité.

Unité $n^{\circ} 3$ : sables et silts

Sur $3,8 \mathrm{~m}$, cette unité se résume en une alternance de zones de sable moyen à grossier et de lits silto-argileux peu épais. On distingue les sous-unités $n^{\text {os }} 3 a$ et $3 b$ : Sous-unité $n^{\circ} 3 a(80 \mathrm{~cm})$ : Cette lentille comprend, à sa base, $45 \mathrm{~cm}$ de sable moyen de couleur grise avec quelques cailloux disséminés. Le sable est suivi d'un lit de cailloux de faible épaisseur et de $35 \mathrm{~cm}$ de sable beige, moyen à grossier, caractérisé par la présence de lits obliques (fig. 4). Des failles inverses sont présentes dans ce sédiment et la direction des chevauchements est vers le SW (1 mesure). Les failles évoquent les contraintes subies par le sable lors de la mise en place des unités glaciaires sus-jacentes. Sous-unité $n^{\circ} 3 b(3 \mathrm{~m})$ : sable moyen à grossier, massif à la base et à granoclassement normal. II est interlité de lits argileux et argilo-silteux vers le sommet. En coupe, certains de ces lits se terminent en biseau. Des cailloux de délestage, peu abondants et de petite dimension (de l'ordre du centimètre), ont été observés. Cette sous-unité s'apparente à un faciès turbiditique.
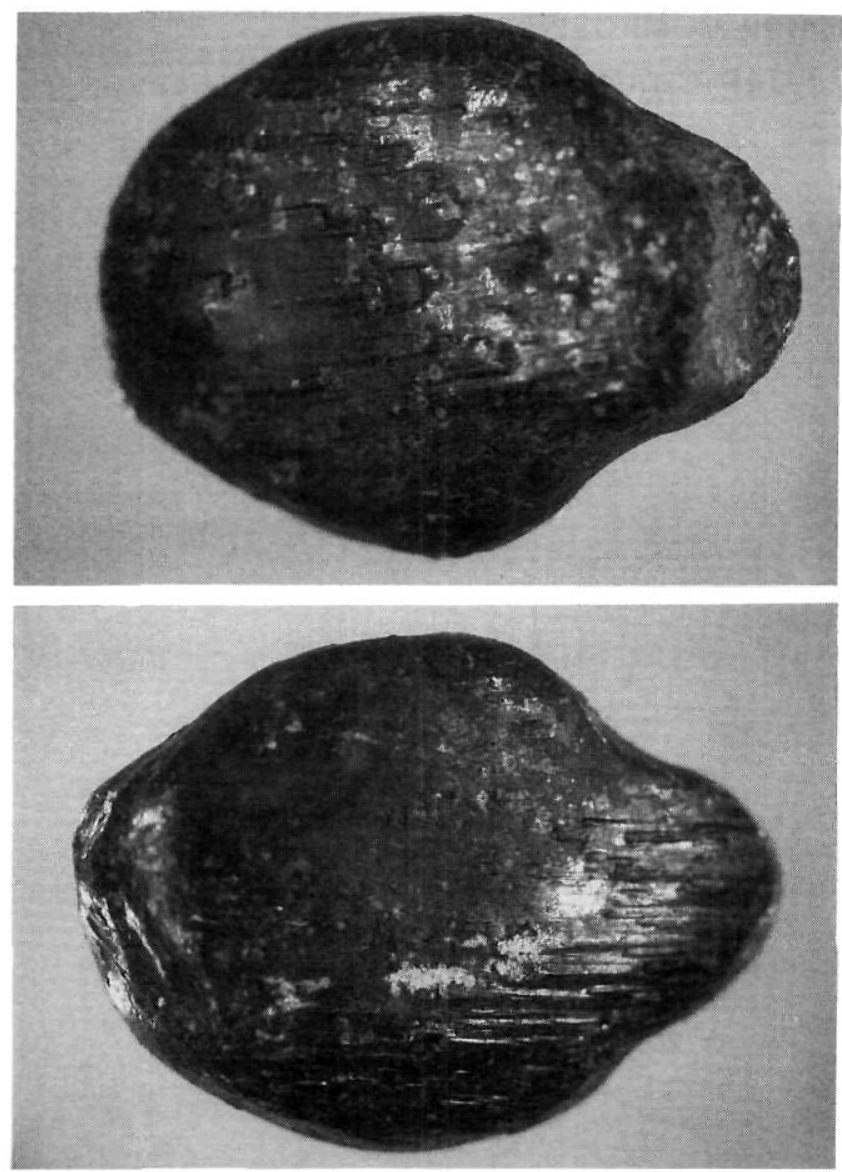

FIGURE 5. Cailloux à queues de rat sur les deux faces ( $a$ et $b$ ) mais de polarité inverse.

Glacial stone with crag-and-tail on both side ( $a$ and b) but of reverse polarity.

Unité $\mathrm{n}^{\circ} 4$ : diamicton II du complexe du Cap Charles

Cette unité, d'une puissance de 3,8 m, est présente sur les trois sites principaux. Elle est mieux exposée à la coupe Tennis 2 , bien qu'on ne puisse pas y observer la base. Ce diamicton de couleur gris foncé est massif, compact et peu carbonaté. Les paramètres granulométriques montrent que la matrice est mal classée et que le pourcentage des particules fines est plus important à la base de l'unité. Le comptage lithologique indique une abondance d'éléments calcaires du Groupe de Trenton $(80 \%)$. La fabrique montre une orientation dominante des cailloux selon l'axe EW. Ceci est confirmé par la présence de cailloux avec queues de rat sur les faces inférieure et supérieure mais de polarité inverse (fig. 5). D'après des mesures in situ, ces queues de rat sont parallèles à la direction de la fabrique du diamicton. L'inclinaison des cailloux vers l'ouest indique un écoulement ou un fluage d'ouest en est. Les queues de rat de polarité inverse ont été vraisemblablement façonnées pendant le transport intraglaciaire dans une zone de cisaillement de la glace. Quelques lits argileux de faible épaisseur sont intercalés à la base du diamicton II, dans une zone de l'ordre de $50 \mathrm{~cm}$ d'épaisseur. Ces lits, non déformés, dont on peut observer les terminaisons en biseau, se sont déposés en conformité à la base du diamicton. Ils indiquent que la sédimentation des lits 
argileux est synchrone avec celle de la partie inférieure du diamicton et, par conséquent, que le diamicton II n'est pas un till de fond mis en place par placage. L'absence d'éléments de lithologie locale, c'est-à-dire de schiste du Groupe d'Utica, est un autre indice tendant à démontrer qu'il ne s'agit pas d'un till de fond. Les caractéristiques du diamicton II s'apparentent à celles des coulées de diamicton (cf. Dreimanis, 1986). Ces coulées de diamicton ont tendance à s'accumuler dans les dépressions, ce qui correspond à la configuration du complexe du Cap Charles (fig. 6b). Selon la nomenclature de Dreimanis et Lundqvist (1984), il s'agirait d'un allo-till, c'est-à-dire d'un till mis en place depuis la surface du glacier. Plus précisément, il est possible que ce sédiment soit un till flué supraglaciaire; le mécanisme de mise en place est analogue aux coulées de diamicton. Boulton (1968) fait état de séquences de tills flués intercalés de lits de sable, de silt et d'argile. Eyles et al. (1988) décrivent aussi des séquences sédimentaires similaires, dans la région de Toronto, mises en place dans un plan d'eau.

Unité $n^{\circ} 5$ : argiles stratifiées

Des argiles massives interstratifiées de quelques minces lits millimétriques argilo-silteux composent l'unité $n^{\circ} 4$. Elles ont une épaisseur de 1,8 m. Les lits argileux ont de 5 à $10 \mathrm{~cm}$ d'épaisseur et sont de couleur gris foncé. Ils se terminent par un pavage de petits cailloux de $2 \mathrm{~cm}$ d'épaisseur. La teneur en carbonates est en moyenne de $8 \%$, avec un maximum de $12,5 \%$ (fig. 2). L'analyse pollinique (Laboratoire de paléobiogéographie et de palynologie à l'Université de Montréal) a révélé que le sédiment est pratiquement stérile $( \pm 1000$ grains $\left./ \mathrm{cm}^{3}\right)$. Le sédiment est dépourvu de microfaune.

TABLEAU I

Description des turbidites de Parisville

\begin{tabular}{|c|c|}
\hline Till de C & entilly \\
\hline $20 \mathrm{~cm}:$ & argile massive \\
\hline $40 \mathrm{~cm}:$ & silt avec structures de traction \\
\hline $40 \mathrm{~cm}:$ & $\begin{array}{l}\text { argile laminée et sable lité affectés par des déformations ( } \mathrm{pa} \\
\text { étirement) }\end{array}$ \\
\hline $20 \mathrm{~cm}:$ & sable et silt stratifiés \\
\hline $55 \mathrm{~cm}:$ & $\begin{array}{l}\text { argile massive luisante avec quelques lits silto-sableux inter } \\
\text { calés. Le tout est déformé au sommet }\end{array}$ \\
\hline $18 \mathrm{~cm}:$ & argile avec lits de sable \\
\hline $60 \mathrm{~cm}:$ & $\begin{array}{l}\text { sable à structure bréchique en alternance avec des lit } \\
\text { silteux }\end{array}$ \\
\hline $20 \mathrm{~cm}:$ & argile luisante tronquée au sommet \\
\hline $50 \mathrm{~cm}$ : & sable diamictique, fissile et compact \\
\hline $50 \mathrm{~cm}:$ & $\begin{array}{l}\text { lits sableux et argileux en alternance avec des passée } \\
\text { diamictiques }\end{array}$ \\
\hline $03 \mathrm{~cm}:$ & diamicton sableux et massif \\
\hline $1,57 \mathrm{~m}:$ & sable turbiditique \\
\hline $28 \mathrm{~cm}:$ & sable diamictique \\
\hline $1,40 \mathrm{~m}:$ & argile massive \\
\hline surface c & d'érosion \\
\hline
\end{tabular}

Unité $\mathrm{n}^{\circ}$ 6: diamicton III du complexe du Cap Charles

Cette unité, d'une puissance de 2,85 m, est bien exposée sur la coupe Tennis 2 . Ce diamicton est massif et compact. II est similaire au diamicton II d'après les données granulométriques, la fabrique, la compaction, la couleur et, dans une moindre importance, la composition lithologique. La matrice est pauvrement classée et le cœfficient d'asymétrie montre une déviation vers les particules fines. La teneur en carbonates de la matrice est en moyenne de $4 \%$. Le comptage lithologique révèle une majorité de cailloux sédimentaires $(60 \%)$, ce qui représente toutefois une baisse substantielle des apports régionaux par rapport au diamicton II. Des lentilles de sable, d'épaisseur décimétrique, sont présentes dans ce sédiment à quelques endroits. Deux plis d'entraînement ont été observés. La poussée qui a engendré ces plis provenait du NE. Ce type de déformation se produit dans un milieu plastique ou semiplastique, selon la teneur en eau (Dreimanis, 1986). La formation de ces plis pourrait également être le résultat du fluage de matériel pendant la mise en place d'un till de fond. La fabrique mesurée montre une orientation des cailloux dans l'axe EW. Compte tenu du mode de mise en place, vraisemblablement par fluage, l'orientation calculée pourrait refléter un fluage secondaire par rapport à l'écoulement général de la glace. Une surface d'érosion caractérise le sommet de cette unité.

\section{Unité $\mathrm{n}^{\circ} 7$ : Les turbidites de Parisville}

D'une épaisseur de $7 \mathrm{~m}$, cette unité est caractérisée par un faciès turbiditique (tabl. I). L'alternance des lits à granulométrie grossière avec des lits argileux laminés correspond aux unités $A$ et $D$ de la séquence de Bouma (1962). Plusieurs lits diamictiques sont intercalés dans cette zone.

\section{Unité $n^{\circ} 8$ : Till de Gentilly}

L'unité en position supérieure au cap Charles est un till de couleur gris pâle à beige. Sa matrice est sableuse, peu compacte et fissile. La teneur en carbonates de la matrice est en moyenne de $6 \%$ et le comptage lithologique montre une prédominance d'éléments sédimentaires du Groupe de Trenton $(80 \%)$ avec un pourcentage de cailloux calcaires altérés dominant. La fabrique indique un écoulement vers le SSW. La description du Till de Gentilly de Occhietti (1982) correspond très bien à cette unité. Cependant, des variations latérales ont été observées. En aval des coupes Tennis 1, 2 et 3 , le faciès du Till de Gentilly repose par-dessus des sables à figures de courant (fig. 6a). Le till devient très épais et atteint environ $11 \mathrm{~m}$. Des intercalations décimétriques de sable ou d'argile silteuse sont présentes et divisent la couche de till en plusieurs lithozones. Certaines de celles-ci sont grossièrement stratifiées. Au sommet, le till est coiffé par une unité argileuse à coquilles marines qui contient de nombreux cailloux. II s'agit probablement d'un sédiment remanié pendant l'exondation au dernier stade de la Mer de Champlain.

\section{INTERPRÉTATION PALÉOENVIRONNEMENTALE}

Le complexe du Cap Charles est intercalé entre deux unités lacıstres, les rythmites de Leclercville et les turbidites de 

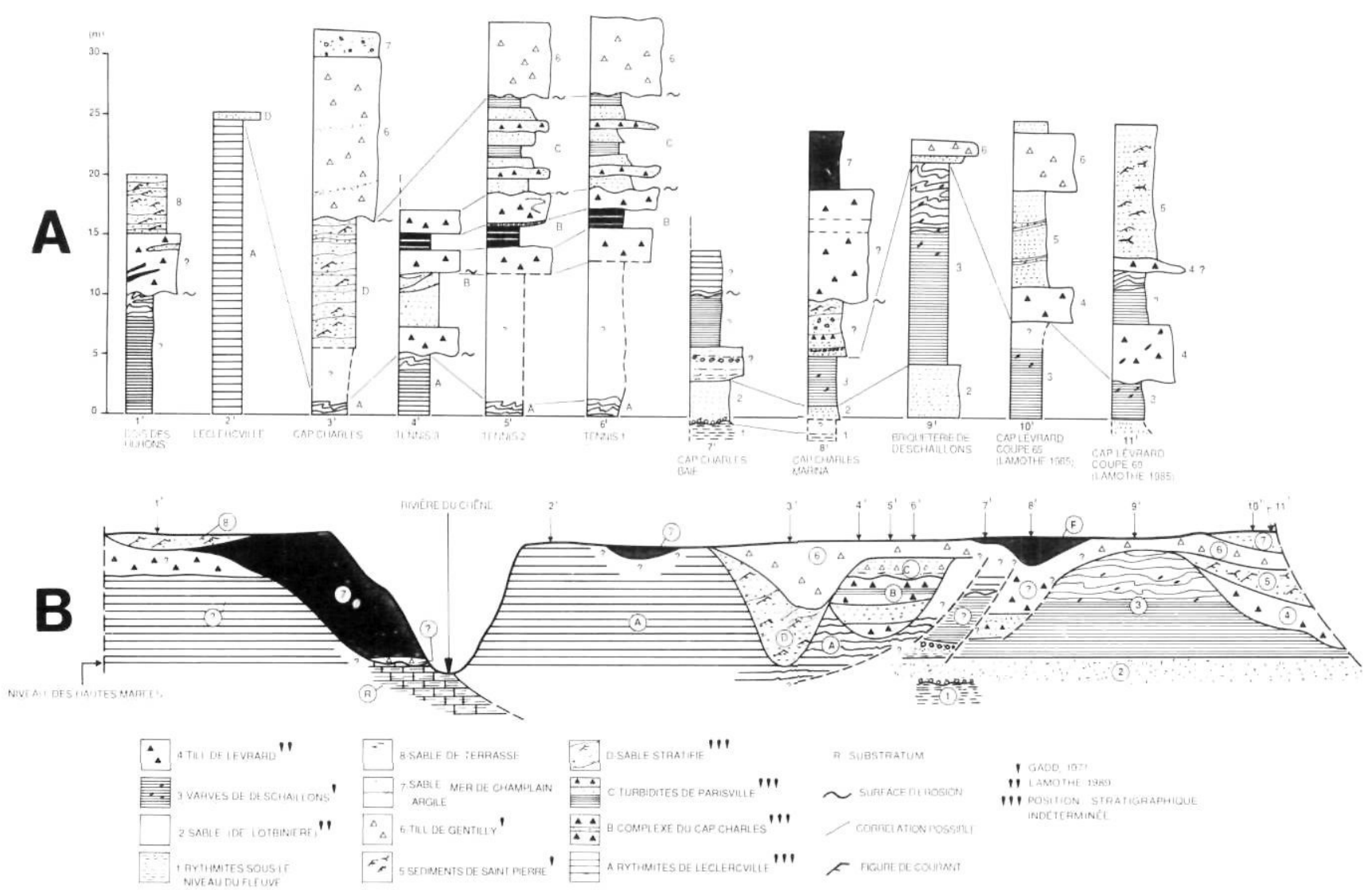

FIGURE 6. Contexte stratigraphique du complexe glaciaire du Cap Charles. A) Colonnes stratigraphiques entre le cap Lévrard et Boisdes-Hurons (voir localisation à la fig. 1). B) Représentation schématique de l'agencement stratigraphique des unités quaternaires sur la rive sud du Saint-Laurent.

Parisville. Ces unités représentent des paléoevironnements distincts.

\section{Les rythmites de Leclercville}

Les rythmites de l'unité $n^{\circ} 1$ correspondent à une ancienne étendue d'eau, apparemment de dimension restreinte et de faible durée. Besré (1989) a compté 294 laminations annuelles sur la coupe exposée à Leclercville. L'origine et la nature du lac restent à préciser.

\section{Le complexe du Cap Charles}

Les diamictons I, II et III représentent trois événements d'origine glaciaire et témoignent de la présence, dans le secteur du cap Charles, d'un glacier et d'un lobe glaciaire adjacent à un plan d'eau. Le faciès sous-glaciaire de l'unité $n^{\circ} 2$ (diamicton I) indique que la glace s'est au moins rendue au site du cap Charles, alors que les caractéristiques des unités $n^{\text {os }} 4$ et 6 évoquent la présence d'une masse de glace à proximité immédiate, mais pas nécessairement à l'emplacement même du cap Charles. Les unités stratifiées $n^{\text {os }} 3$ et 5 ont été déposées dans un milieu de marge glaciaire. Les sables de l'unité $n^{\circ} 3$ témoignent d'une énergie relativement importante, alors que l'unité $n^{\circ} 5$, argileuse, représente une position plus distale de
Stratigraphic setting of the Cap Charles glacial context. A) Sections along the south shore of the St. Lawrence River between the cap Lévrard and Bois-des-Hurons (see Fig. 1 for location). B) Schematic representation of the stratigraphy of the Quaternary units on the south shore of the St. Lawrence River. 
posent ne sont pas indicateurs de changements climatiques majeurs. II est possible que le faciès glaciaire du cap Charles corresponde à un événement relativement court et localisé. Nous pourrions alors envisager la présence d'un lobe glaciaire pour expliquer sa mise en place, sur le même modèle que les lobes de la région des Grands Lacs (Karrow, 1984).

\section{POSITION STRATIGRAPHIQUE DU COMPLEXE DU CAP CHARLES ET CHRONOLOGIE DES ÉVÉNEMENTS}

Dans la région étudiée, plusieurs surfaces d'érosion ont été observées dans les séquences sédimentaires; elles sont les témoins de l'emboîtement de corps sédimentaires dans des séquences plus anciennes. Les levés de terrain de cette étude montrent plus précisément que chaque corps sédimentaire glaciaire, pris dans le sens d'une nappe régionale de la même unité, est composé en fait de nombreuses lentilles de faible largeur $(n=100)$ et d'épaisseur variable emboîtées dans diverses unités sous-jacentes. Les lentilles peuvent également montrer de fortes variations latérales de faciès et une superposition de lithozones qui varie localement. Ce modèle lithostratigraphique s'applique probablement à chaque épisode glaciaire de la région. À titre d'exemple, la figure $6 \mathrm{~b}$ montre l'emboîtement du complexe du Cap Charles dans les rythmites de Leclercville, le tout vraisemblablement emboîté dans une séquence plus ancienne composée des Varves de Deschaillons, du sable sous-jacent et des rythmites du fleuve. La position des rythmites de Leclercville n'est pas encore définitivement démontrée. Elles sont vraisemblablement postérieures aux Varves de Deschaillons. Les rythmites à la base de la coupe du cap Charles sont corrélées aux rythmites de Leclercville en raison du contenu pollinique similaire et du comptage des couplets saisonniers sur les zones exposées au cap Charles et à Leclercville (Besré, 1989). Du point de vue stratigraphique, trois hypothèses principales (fig. 7) peuvent être proposées sur la position et l'âge relatif du complexe du Cap Charles par rapport à la surface d'érosion qui le tronque. Selon la première hypothèse, la surface d'érosion ne représente pas une lacune de sédimentation importante et l'ensemble du complexe représente un membre inférieur de la formation incluant les turbidites de Parisville et le Till de Gentilly. Dans cette éventualité, les Sédiments de Saint-Pierre sensu stricto, qui n'affleurent pas au site étudié, sont antérieurs au complexe du Cap Charles. Le complexe du Cap Charles serait alors plus jeune que $75 \mathrm{ka}$ (âge au ${ }^{14} \mathrm{C}$, Stuiver et al., 1978) ou que 60 ka (âge obtenu par thermoluminescence, Lamothe et Huntley, 1988). II marquerait le début de l'englaciation majeure du Stade de Trois-Rivières. La deuxième hypothèse repose sur la présomption que la lacune de sédimentation située au-dessus de l'unité $n^{\circ} 6$ pourrait correspondre à l'épisode de l'intervalle non glaciaire de Saint-Pierre sensu stricto. Le complexe du Cap Charles serait alors associé aux tills directement antérieurs aux Sédiments de Saint-Pierre dans la vallée du Saint-Laurent. Une troisième éventualité, moins plausible, associerait le complexe du Cap Charles à une phase glaciaire distincte, plus jeune que les tills antérieurs aux Sédiments de Saint-Pierre et plus vieille que le Till de Gentilly.

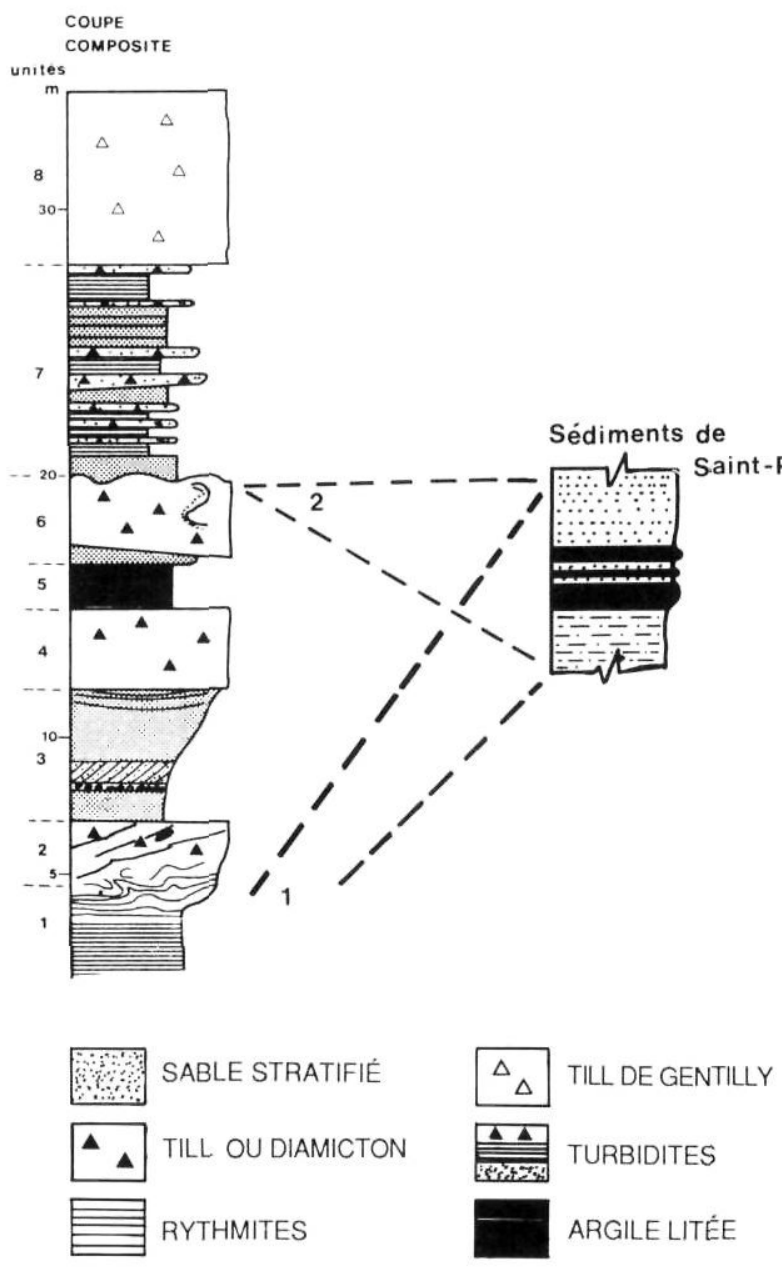

FIGURE 7. Deux hypothèses sur la position stratigraphique du complexe du Cap Charles par rapport aux Sédiments de Saint-Pierre.

Two stratigraphic hypotheses for the position of the Cap Charles glacial complex compared with the St. Pierre Sediments position.

\section{CONCLUSION}

Le complexe du Cap Charles représente une nouvelle unité lithostratigraphique d'origine glaciaire, antérieure au Till de Gentilly. Il est composé de trois zones de diamicton séparées par des sables ou des argiles stratifiées. II est intercalé entre les rythmites de Leclercville, dont la position stratigraphique n'est pas connue, et les turbidites de Parisville qui précèdent directement le Till de Gentilly. II représente un épisode d'avancée glaciaire vers le SE suivi apparemment de fluage de till en milieu glaciolacustre. Faute de repères, la position stratigraphique du complexe du Cap Charles n'est pas définie. II peut représenter une phase préliminaire à l'englacement généralisé du Till de Gentilly ou un épisode glaciaire distinct antérieur à cet englacement généralisé. II peut également représenter un épisode glaciaire court en relation avec les tills immédiatement antérieurs aux Sédiments de Saint-Pierre. Le complexe du Cap Charles implique que le modèle stratigraphique des unités glaciaires de la vallée moyenne du SaintLaurent est plus complexe que le schéma traditionnel (Gadd, 1971) ou que le schéma révisé par Lamothe $(1985,1987)$. 


\section{REMERCIEMENTS}

Ce travail a été réalisé grâce à une bourse d'étude du Fonds pour la formation de chercheurs et l'aide à la recherche du ministère de l'Éducation du Québec et à une subvention du Conseil de recherche en sciences naturelles et génie du Canada. Nous remercions le Département des sciences de la Terre de l'Université du Québec à Montréal et particulièrement M. Marc Durand pour ses conseils, M. Robert-André Daigneault pour sa coopération en laboratoire et $\mathrm{M}^{\mathrm{me}}$ Michelle Laithier pour les travaux graphiques. L'aide de $\mathrm{M}^{\mathrm{me}}$ Mireille Bouchard et de M. André Parent du Département de géographie de I'UQAM, et de $\mathrm{M}^{\mathrm{me}}$ Marion Lepage fut grandement appréciée. Nous tenons à remercier également MM. Pierre Ferland et François Besré pour leurs judicieux conseils, leur appui moral et l'organisation matérielle, Michel Parent pour ses suggestions pertinentes et Christophe Rogier pour son aide sur le terrain. Nous sommes aussi reconnaissants de l'accueil chaleureux de M. Duhamel au cap Charles.

\section{RÉFÉRENCES}

Besré, F., 1989. Les Varves de Deschaillons, les Rythmites du SaintMaurice et les rythmites de Leclercville du Pléistocène supérieur de la vallée du Saint-Laurent. Thèse de M.Sc., Université du Québec à Montréal, $83 \mathrm{p}$.

Bouma, A. H., 1962. Sedimentology of some flysch deposits: a graphic approach to facies interpretation. Elsevier, Amsterdam, $168 \mathrm{p}$.

Boulton, G. S., 1968. Flowtills and related deposits on some Vestspitsbergen glaciers. Journal of Glaciology, 7 (51): 391-412.

1970a. On the origin and transport of englacial debris in Svalbard glaciers. Journal of Glaciology, 9 (56) : 213-229.

1970b. On the deposition of subglacial and melt-out tills at the margins of certain Svalbard glaciers. Journal of Glaciology, 9 (56) : 231-245.

1976. The origin of glacially fluted surfaces-observations and theory. Journal of Glaciology, 17 (76) : 287-309.

Clark, T. H. et Globensky, Y., 1975. Rapport géologique, région de Grondines. Ministère des Richesses naturelles, Québec. R-G-154.

Dreimanis, A., 1976. Tills: their origin and properties, p. 11-14. In R. F. Legget, édit. Royal Society of Canada, Special Publication $\mathrm{n}^{\circ} 12$.

1985. Commission on genesis and lithology of Quaternary deposits (INQUA), circulaire $\mathrm{n}^{\circ} 26$.

1986. Commission on genesis and lithology of Quaternary deposits (INQUA), circulaire $\mathrm{n}^{\circ} 28$.

Dreimanis, A. et Lundqvist, J., 1984. What should be called till? Striae, 20: $5-10$

Eyles, N., Eyles, C. H. and Miall, A. D., 1983. Lithofacies types and vertical profile models; an alternative approach to the description and environmental interpretation of glacial diamict and diamictite. Sedimentology, 30: 393-410.

Eyles, N. et Clark, B. M., 1988. Last interglacial sediments of the Don Valley Brickyard, Toronto, Canada, and their paleoenvironmental significance. Canadian Journal of Earth Sciences, 25; 1108-1122.

Folk, R. L. et Ward, W. C., 1957. Brazos river bar: a study in significance of grain size parameters. Journal of Sedimentary Petrology, $27(1): 3-26$.
Gadd, N. R., 1971. Pleistocene geology of the central St. Lawrence Lowland, with selected passage from an unpublished manuscript: The St. Lawrence Lowland, by J. W. Goldthwait. Geological Survey of Canada, Memoir 359, 153 p.

Hedberg, H., 1979. Guide stratigraphique international, classification, terminologie et règles de procédures. Doin, Paris, $233 \mathrm{p}$.

Karrow, P. F., 1957. Pleistocene geology of the Grondines map-area, Québec. Thèse de Ph.D., University of Illinois, Urbana, $97 \mathrm{p}$.

1984. Quaternary stratigraphy and history, Great Lakes-St. Lawrence region, p. 137-153. In R. J. Fulton, édit., Quaternary stratigraphy of Canada - A Canadian Contribution to IGCP Project 24. Geological Survey of Canada, Paper 84-10.

Keele, J., 1915. Preliminary report on the clay and shale deposits of the Province of Québec. Geological Survey of Canada, Memoir 64, $175 \mathrm{p}$.

Lamothe, M., 1985. Lithostratigraphy and geochronology of the Quaternary deposits of the Pierreville and St-Pierre-les-Becquets areas, Québec. Thèse de Ph.D., University of Western Ontario, London, $227 \mathrm{p}$.

1987. Pleistocene stratigraphy in the St. Lawrence Lowlands and the Appalachians of southern Québec, a field guide. Collection Environnement et Géologie, vol. 4, Université de Montréal, 210 p.

1989. Stratigraphie du Pléistocène de la région de Saint-Pierreles-Becquets, p. 23-34. In M. Lamothe édit., Stratigraphie du Pléistocène du Québec méridional. Réunion annuelle de l'Association géologique du Canada, excursion A1, $118 \mathrm{p}$.

Lamothe, M. et Huntley, D. J., 1988. Thermoluminescence dating of late Pleistocene sediments. St. Lawrence Lowlands, Québec. Géographie physique et Quaternaire, 42: 33-44.

McEachren, D. B., 1986. Stereo, the stereographic projection programme. Apple Macintosh computer, version 1.3.

Occhietti, S., 1982. Synthèse lithostratigraphique et paléoenvironnements du Quaternaire au Québec méridional. Hypothèse d'un centre d'englacement wisconsinien au Nouveau-Québec. Géographie physique et Quaternaire, 36: 15-49.

1987. Dynamique de l'Inlandsis laurentidien du Sangamonien à l'Holocène. Géographie physique et Quaternaire, 41: 301-315.

Occhietti, S., Clet, M., Bernier, F., Besré, F., Ferland, P. et Lancery, J.-M., 1989. Contribution à la lithostratigraphie de la vallée moyenne du Saint-Laurent: Saint-Pierre-les-Becquets, Sainte-Anne-de-LaPérade et Donnacona, p. 35-53. In M. Lamothe, édit., Stratigraphie du Pléistocène du Québec méridional. Réunion annuelle de l'Association géologique du Canada, excursion A1, $118 \mathrm{p}$.

Parent, M., 1987. Late Pleistocene stratigraphy and events in the Asbestos-Valcourt region, southeastern Québec. Thèse de Ph.D., University of Western Ontario, London, $292 \mathrm{p}$.

Simard, L., 1971. Relevé des caractéristiques des roches et dépôts meubles de la vallée du Saint-Laurent. Ministère des Travaux publics, Division du chenal maritime du Saint-Laurent, Montréal, 1. $331 \mathrm{p}$.

Stuiver, M., Heusser, C. J. and Yang, I. C., 1978. North American glacial history extended to 75,000 years ago. Science, 200: 16-21.

Terasmae, J., 1958. Contribution to Canadian palynology, pt. 2; nonglacial deposit in the St. Lawrence Lowlands, Québec. Geological Survey of Canada, Bulletin 46: 13-28. 